\title{
A ESTIMULAÇÃO MAMÁRIA COMO UM MÉTODO NATURAL DE INDUÇÃO DO TRABALHO DE PARTO
}

\author{
${ }^{1}$ Filipa Ramos, ${ }^{2}$ Emília Coutinho e ${ }^{3}$ Vitória Parreira \\ ${ }^{1}$ Saúde Materna e Obstétrica no CHTS, Portugal. filipa.mota.ramos@gmail.com \\ 2 Escola Superior de Saúde de Viseu, Portugal. ecoutinhoessv@gmail.com \\ ${ }^{3}$ Escola superior de Enfermagem do Porto, Portugal. - vitoria@esenf.pt
}

\begin{abstract}
Resumo. A OMS define Parto Normal aquele que apresenta início espontâneo, baixo risco desde o início até ao nascimento, excluindo mulheres submetidas a indução de trabalho de parto (TP). A estimulação mamária (EM) tem sido historicamente utilizada como método não farmacológico, natural de indução do TP; Objetivo: Conhecer a eficácia e a segurança da estimulação mamária enquanto método de indução natural do TP Métodos: Revisão Integrativa da Literatura; Resultados: Outcomes eficácia: scores de índice de Bishop significativamente melhores, tendência para início do TP espontâneo em 72h, menores níveis de cesariana e níveis de satisfação excelentes; Outcomes segurança: não existiram diferenças significativas relativamente à presença de mecónio, APGAR $<7$ aos 5 minutos e cesariana em comparação com grupos de controlo (sem intervenção) ausência de casos de hiperestimulação uterina; Conclusões: A EM mostra-se segura e eficaz, sendo razoável considerá-la como opção viável para induzir o TP de baixo risco.
\end{abstract}

Palavras-chave: Enfermagem Saúde Materna e Obstétrica; Trabalho de Parto, Parto Normal; Estimulação Física; Mama.

\section{BREAST STIMULATION AS NATURAL METHOD TO INDUCING NORMAL LABOR}

Abstract. WHO defines Normal Childbirth as the one that presents spontaneous onset, low risk at the beginning until birth, excluding women submitted to induction of labor. Breast stimulation (BS) has historically been used as a non-pharmacological, natural method of inducing and accelerating labor; Goals: Understanding the efficacy and safety of BS as a natural method of inducing labor; Methods: Integrative Literature Review; Results: Outcomes effectiveness: scores of Bishop significantly better, a tendency to spontaneous labor oncet in 72 hours, lower levels of C-section and great satisfaction levels; Safety outcomes: there were no significant differences in the presence of meconium, APGAR $<7$ at $5 \mathrm{~min}$ and $\mathrm{C}$ - section compared to control groups (without intervention) absence of cases of uterine hyperstimulation; Conclusions: BS is a safe and effective method, and it is reasonable to consider as a viable option to induce low-risk labor.

Keywords: Nurse Midwives; Obstetric Labor; Natural Childbirth; Physical Stimulation; Breast.

\section{INTRODUÇÃO}

A opção pelo parto normal atravessa tempos e culturas. Todavia, sob a égide do avanço técnico científico mudaram os rituais do parto e do nascimento ficando à mercê de uma prática biomédica, intervencionista, em que a indução do trabalho de parto é suportada em métodos farmacológicos. Ora se por um lado é garantida a eficácia, por outro, perde se a essência do parto normal que hoje muitas mulheres e casais voltam a reclamar como opção e como um direito. 
Segundo a Organização Mundial de Saúde (OMS, 2018), 85 a 90\% de todos os nascimentos dão-se sem fatores de risco e sem complicações. Esta emanou recomendações que além dos requisitos clínicos, atendem às necessidades psicológicas e emocionais das mulheres, defendendo o parto normal. Este é definido como o que apresenta início espontâneo, de baixo risco desde o início até ao nascimento. A criança nasce espontaneamente, em apresentação cefálica de vértice, entre as 37 e as 42 semanas completas de gravidez, posteriormente ao parto a mãe e o RN apresentam-se em boa condição. Excluídos desta definição encontram-se os partos resultantes de indução de trabalho de parto com prostaglandinas, ocitócicos ou rotura artificial de membranas (OMS, 2018). Foi neste sentido, que nos propusemos atingir o objetivo de conhecer a eficácia e segurança da estimulação mamária enquanto método de indução natural do trabalho de parto de baixo risco.

Os protocolos vigentes da indução do TP, deliberados pela decisão médica, a mulher apresenta um papel passivo e assentam numa abordagem ativa e interventiva, com o recurso a medidas farmacológicas com elevado risco de complicações, como a hemorragia peri-parto, cesariana, hiperestimulação uterina, assim como a menores níveis de satisfação materna (NICE, 2018; OMS, 2018). São, ainda, critérios para a indução de trabalho de parto as situações de complicações materno-fetais que impliquem o termino da gravidez (ex.: préeclâmpsia) e a gravidez saudável com 41 semanas, critério com baixo nível de evidência científica (OMS, 2018).

O recurso a métodos não farmacológicos e naturais para a indução do TP não é uma prática desconhecida, muitos métodos têm sido utilizados desde há muitos anos em diferentes culturas. A estimulação mamária (EM) tem sido historicamente utilizada para induzir o TP; textos médicos europeus oriundos do séc. XVIII reportam o recurso à estimulação mamária na gestão do parto pós-datado. Hoje, nomeadamente na India e na cultura japonesa $50 \%$ das mulheres recorre a este método e ao exercício físico para ajudar a induzir o trabalho de parto (Demirel \& Guler, 2015; Kavanagh, Kelly \& Thomas 2005; Takahata et.al, 2018). Corroborando este facto, a ACOG (2019) e a NICE (2018) reconhecem o recurso à EM como método não farmacológico de Indução de TP.

Diversos mecanismos encontram-se na base do início do TP associados, por sua vez, a múltiplos fatores, entre os quais o nível de ocitocina libertada pela glândula pituitária. Esta hormona tem um papel preponderante enquanto estimuladora da contratilidade do útero. $A$ 
base para o mecanismo da EM baseia-se na produção de ocitocina endógena através da estimulação da mama/mamilo, semelhante ao mecanismo existente na amamentação. Durante a gravidez e antes do início do TP, os níveis de oxitocina mantêm-se estáveis. No entanto, com estimulação da produção e aumento da sua concentração, a parede uterina torna-se mais sensível à sua ação, dando-se início ao TP. Sabe-se que a libertação de ocitocina endógena leva à maturação uterina (melhora, portanto, o índice de Bishop) e que as contrações uterinas, por sua vez, levam ao início de trabalho de parto espontâneo e natural.

A definição de EM não é clara, no entanto, esta pode ser definida como a autoestimulação através da massagem manual da mama/mamilo ou através da sucção mecânica por bombas de extração de leite (Demirel \& Guler, 2015; Kavanagh, Kelly \& Thomas 2005; Takahata et.al, 2018).

A EM, como meio de indução do trabalho de parto, pode oferecer à mulher maior controlo sobre o processo de trabalho de parto e, ainda, apresentar a vantagem de ser natural, fisiológico, um método não dispendioso, não doloroso e promotor do parto normal (Kavanagh, Kelly e Thomas 2005; Takahata et.al, 2018).

Parte-se do pressuposto que para a excelência do exercício profissional é essencial o recurso a uma prática baseada na evidência. Neste contexto, os enfermeiros especialistas em saúde materna e obstétrica cujos cuidados especializados que prestam se centram na mulher, devem ser fomentadores de práticas que sustentem o parto normal. com a menor intervenção obstétrica possível, que seja essencial à procura e ao desenvolvimento de conhecimentos de métodos naturais e fisiológicos que otimizem o trabalho de parto e potenciem a capacitação da mulher ao longo de todo o processo, nomeadamente no que toca à oferta de alternativas ao processo tradicional de indução do TP.

\section{METODOLOGIA}

Pela sua pertinência e característica adotou-se como metodologia a revisão integrativa da literatura como suporte da prática baseada na evidência. Existe um aumento considerável de produção de investigação sustentada em revisões, sejam elas sistemáticas, metaanálises ou revisões integrativas. 
Esta comporta-se como a única abordagem que permite a integração e a combinação de diversas metodologias, desde estudos experimentais ou não experimentais do foro qualitativo, permitindo, deste modo, ter um conhecimento mais abrangente sobre um determinado fenómeno em estudo e tornando-se mais relevante para a Enfermagem (Sousa, Vieira, Severino \& Antunes, 2017; Whittemore \& Knafl, 2005). A variedade na composição da amostra na revisão integrativa em conjunção com a multiplicidade de finalidades deste método proporciona como resultado um quadro completo de conceitos, teorias ou problemas relativos ao cuidado na saúde, relevantes para a enfermagem (Mendes, Silveira \& Galvão, 2008, p. 780).

A RIL é, assim, um método de investigação que permite a procura, a avaliação crítica e síntese de evidências existentes sobre um determinado tema, onde o produto final é o estado de conhecimento do tema investigado. Por outro lado, ainda permite detetar fragilidades, conduzindo ao desenvolvimento de novas investigações (Sousa et al., 2017, p. 20).

Para a construção deste estudo procedemos à operacionalização das seis fases que é preconizada pela revisão integrativa da literatura: (1) identificação do tema e seleção da hipótese ou questão de pesquisa; (2) determinação de critérios de inclusão e exclusão de estudos selecionados ou pesquisa da literatura (3) definição das informações a serem extraídas dos estudos selecionados/categorização dos estudos; (4) avaliação dos estudos incluídos; (5) interpretação dos resultados e, (6) apresentação da revisão/síntese de conhecimentos (Sousa et al., 2017).

Tendo por base o problema em estudo, relacionado com uma intervenção de enfermagem e o seu impacto no trabalho de parto e a sua influência na mulher, este processo metodológico permite-nos sintetizar achados sobre este fenómeno e obter uma visão ampla sobre o conhecimento até agora existente sobre esta temática.

Por conseguinte, definimos como objetivo para esta investigação: conhecer a eficácia e a segurança da estimulação mamária, enquanto método de indução natural do trabalho de parto. Neste sentido, para podermos guiar a investigação e formular a questão de partida, recorremos à estratégia PICO. Esta traduz quatro elementos que se mostram basais para a condução da pesquisa: Pessoa/problema; a Intervenção; Comparação/controlo; e Outcomes/resultados. 
Em concreto, a população a ser estudada foi constituída por grávidas de baixo risco e de termo. A intervenção foi a estimulação mamária em comparação com placebo (ou sem estimulação) ou outro método (ex. indução sintética). Pretendemos obter resultados relacionados com a eficácia e segurança para parturiente e feto enquanto método nãofarmacológico de indução do TP. Neste sentido surgiu a questão de investigação: "Qual a eficácia e segurança da estimulação mamária enquanto técnica não-farmacológica, natural, de indução do trabalho de parto em grávidas de baixo risco e de termo?". As Estratégias de pesquisa da literatura bem definidas são essenciais, para aprimorar o rigor de qualquer tipo de revisão, já que pesquisas incompletas e tendenciosas resultam no potencial de resultados imprecisos (Whittemore \& Knafl 2005). Por conseguinte, para termos de pesquisa, utilizamos as palavras-chave, com termos $\mathrm{MesH}$, combinados com operadores boleanos: "breast OR nipple Stimulation" "indution AND Labor". Recorremos às bases de dados: CINAHL Complete, MEDLINE Complete, Nursing \& Allied Health Collection, Cochrane Database of Systematic Reviews, Cochrane Methodology Register, Library, Information Science \& Technology Abstracts, MedicLatina e Clinicalkey.

Definimos como critérios de inclusão os estudos publicados nas bases de dados entre 2015 e 2019, apresentados nos idiomas de inglês, francês ou português, e cujo título e/ ou resumo fizessem referência à temática. Todavia, deparámo-nos com um número muito reduzido de artigos, pelo que se impôs a necessidade em alargar o intervalo de tempo, em anos na pesquisa, de 2010 até 2019. Obtivemos um total de 10 artigos. Estabelecemos como critérios de exclusão: - estudos que não apresentassem texto completo e/ou se apresentassem repetidos, tendo obtido um total final de 6 estudos (Figura 1). Face à existência de um estudo revisão sistemática $\operatorname{Cochrane}^{\circledR}$ de 2005, consideramos pertinente a sua inclusão pela sua relevância para a evidência científica, pelo que se obteve um total final de 7 artigos (Figura 1).

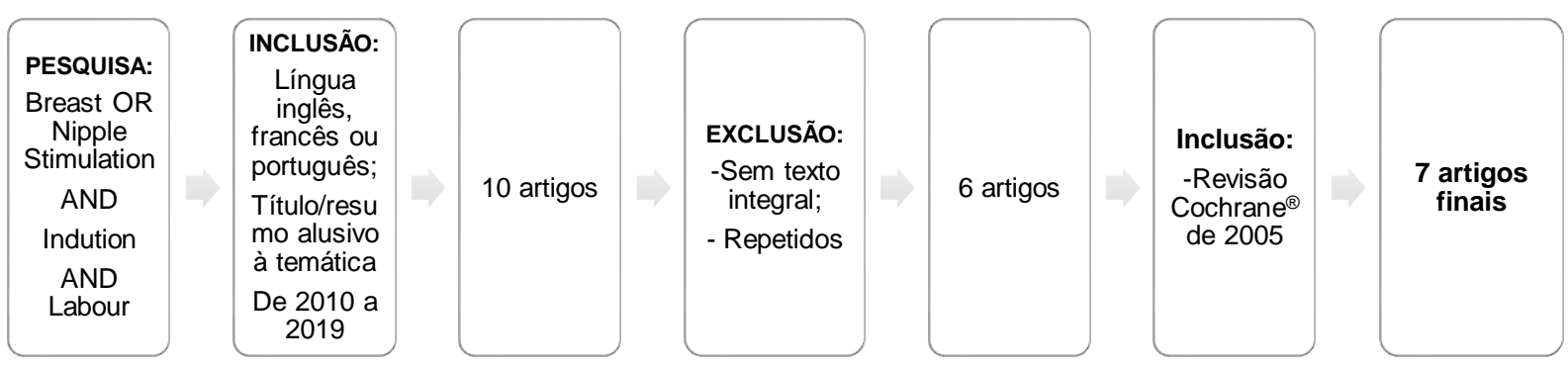

Figura 1 - Condução da pesquisa: seleção de artigos 
Após a identificação e seleção dos artigos, procedeu-se à definição das informações a serem extraídas dos estudos selecionados e à categorização dos mesmos. Foram extraídos dados dos estudos relativos à abordagem, contexto, amostra e principais conclusões. Os ensaios foram, categorizados quanto ao nível de evidência, tendo para isso por base a classificação de Oxford Centre for Evidence-Based Medicine. Esta classificação permitiu identificar o perfil dos estudos desenvolvidos sobre este tema.

Quadro 1 - Estudos selecionados

\begin{tabular}{|c|l|}
\hline 1 & Kavanagh J; Kelly AJ; Thomas J (2005) Breast stimulation for cervical ripening and induction of labour \\
\hline 2 & $\begin{array}{l}\text { Ellen J. Razgaitis, Ashlee N. Lyvers (2010) Management of Protracted Active Labor with Nipple } \\
\text { Stimulation: A Viable Tool for Midwives? }\end{array}$ \\
\hline 3 & $\begin{array}{l}\text { Singh N, Tripathi R, Mala YM \& Yedla N (2014) Breast Stimulation in Low-Risk Primigravidas at Term: } \\
\text { Does It Aid in Spontaneous Onset of Labour and Vaginal Delivery? A Pilot Study }\end{array}$ \\
\hline 4 & $\begin{array}{l}\text { Demirel G.; Guler H.(2015) The Effect of Uterine and Nipple Stimulation on Induction With Oxytocin } \\
\text { and the Labor Process }\end{array}$ \\
\hline 5 & $\begin{array}{l}\text { Suja J..(2015) Study To Evaluate The Effectiveness of Nipple Stimulation For Progress of Labour } \\
\text { During First Stage, Among Primigravida Mothers in Selected Hospital at Tirunelveli.. }\end{array}$ \\
\hline 6 & $\begin{array}{l}\text { Takahata K, Horiuchi S, Tadokoro Y, Shuo T, Sawano E \& Shinohara K.(2018) Effects of breast } \\
\text { stimulation for spontaneous onset of labor on salivary oxytocin levels in low-risk pregnant women: A } \\
\text { feasibility study. }\end{array}$ \\
\hline 7 & $\begin{array}{l}\text { Suresh, V.; Soni, r. (2019) A Quasi Experimental Study to Assess the Effectiveness of Nipple } \\
\text { Stimulation in the Progress of Labour among Primipara Women during First Stage of Labour in } \\
\text { Selected Hospital of Jabalpur (M.P.) }\end{array}$ \\
\hline
\end{tabular}

Sabemos que, quando metodologicamente estão incluídas diversas fontes primárias aumenta a complexidade da avaliação de dados (Whittemore \& Knafl 2005).

Assim, respeitamos o rigor metodológico exigido, inerente à análise cuidadosa de cada estudo.

Os estudos selecionados apresentaram evidencias de nível I a III: estudos de revisão $(n=2)$, randomizados controlados $(n=2)$ e quase-experimentais $(n=3)$; abordagem qualitativa $(n=1)$. Estes foram realizados na sua maioria na área de enfermagem $(n=6)$ tendo a revisão sistemática abordado áreas da medicina, demonstrando a pertinência multidisciplinar do tema. Em relação à origem dos estudos estes foram oriundos da India $(n=3)$, Reino Unido, Turquia, Japão e EUA. Todos os artigos estavam escritos na língua inglesa.

Contribuição dos autores do presente trabalho: $01^{\circ}$ e $3^{\circ}$ autores trabalharam na conceção, execução, análise, interpretação e construção da investigação e do artigo. $\bigcirc 2^{\circ}$ autor contribuiu para a construção do artigo. 


\section{RESULTADOS E DISCUSSÃO}

Visando a sistematização dos dados, desenvolvemos um instrumento de representação gráfica, tabelar que estruturasse a informação relativa aos dados dos autores, publicação (local, data), nível de evidência, resultados e principais conclusões obtidas centradas na eficácia e segurança da técnica, que se apresentam no quadro seguinte (Quadro 2).

Quadro 2 - Categorização e informações dos estudos selecionados

\section{Breast stimulation for cervical ripening and induction of labor}

N.E.

Kavanagh J; Kelly AJ; Thomas J; Cochrane Collaboration ${ }^{\circledR} ; 2005$, Reino Unido

\begin{tabular}{|c|c|}
\hline$\underset{⿱ 亠 䒑}{\stackrel{D}{\Sigma}}$ & $\begin{array}{l}\text { isão sistemática com meta-análise de ensaios clínicos randomizados. } \\
\text { isão e Análise de RTC's até } 2005 \text { (com revisão em 2010) Inclusão de } 6 \text { estudos - } \\
\text { de } 719 \text { mulheres }\end{array}$ \\
\hline & $\begin{array}{l}\text { Outcome eficácia apresenta ter menor número de mulheres com necessidade de } \\
\text { cesariana em comparação a nenhuma intervenção ou uso de oxitocina, apesar da } \\
\text { diferença não ser estatisticamente significativa no último caso. } \\
\text { A diferença de mulheres sem início de trabalho de parto em } 72 \mathrm{~h} \text { foi estatisticamente } \\
\text { significativa (menor no grupo EM). Relativamente ao uso de oxitocina, esta diferença } \\
\text { não se mostrou significativa (menor no grupo da oxitocina) } \\
\text { Outcome segurança: Menor rácio de mecónio à nascença em comparação a sem } \\
\text { intervenção ou uso de oxitocina, mas não estatisticamente significativo. } \\
\text { A estimulação mamária parece ser benéfica relativamente à redução do número de } \\
\text { mulheres em não trabalho de parto após } 72 \text { horas e reduz as taxas de hemorragia pós- } \\
\text { parto. }\end{array}$ \\
\hline
\end{tabular}

Management of Protracted Active Labor with Nipple Stimulation: A Viable Tool for Midwives?

Ellen J. Razgaitis, Ashlee N. Lyvers; American College of Nurse Midwives- Journal Midwifery Women's Health; 2010, EUA: New York City.

\begin{tabular}{|c|c|}
\hline $\begin{array}{l}\mathbf{D} \\
\Sigma\end{array}$ & $\begin{array}{l}\text { Revisão da literatura } \\
\text { Abrangidos } 5 \text { estudos. Selecionados } 2 \text { (N=163 grávidas) }\end{array}$ \\
\hline 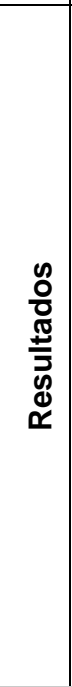 & $\begin{array}{l}\text { Eficácia: não houve diferença estatisticamente significativa relativamente à duração do } \\
\text { primeiro estadio do trabalho de parto nos grupos de estimulação mamilar e ocitocina; } \\
\text { Outocome Segurança: os estudos explorados um dos estudos relatou zero casos de } \\
\text { hiperestimulação uterina entre as mulheres que usaram a EM/Ocitocina, no outro a } \\
\text { hiperestimulação não foi relatada separadamente de outros eventos adversos, pelo que, } \\
\text { a taxa de ocorrência nesse estudo é desconhecida. } \\
\text { Não foram encontradas diferenças estatisticamente significativas entre os grupos em } \\
\text { termos de taxa de parto cesariana e pH da artéria umbilical. Apesar de não ser } \\
\text { estatisticamente significativo, as mulheres que usaram estimulação de mamilo e } \\
\text { ocitocina tiveram maiores rácios de cesariana, seguindo-se do grupo de ocitocina e por } \\
\text { fim no grupo de estimulação de mamilo com menor rácio. Nenhuma diferença } \\
\text { significativa nos scores de Apgar em } 1 \text { e } 5 \text { minutos entre os métodos de estimulação do } \\
\text { mamilo apenas e ocitocina apenas. Embora esses dados sejam limitados, eles sugerem } \\
\text { que a estimulação do mamilo não coloca o feto em risco aumentado de desfechos } \\
\text { adversos. } \\
\text { Apesar da pesquisa limitada sobre a eficácia, a estimulação do mamilo parece ser } \\
\text { segura, já que não houve eventos adversos nos estudos revistos.. }\end{array}$ \\
\hline
\end{tabular}


Breast Stimulation in Low-Risk Primigravidas at Term: Does It Aid in Spontaneous Onset of Labour and Vaginal Delivery? A Pilot Study

The Effect of Uterine and Nipple Stimulation on Induction With Oxytocin and the Labor Process

Demirel G. ; Guler H.; Worldviews on Evidence-Based Nursing; 12:5, 273-280; 2015, Turquia

\begin{tabular}{|c|c|}
\hline$\frac{\grave{8}}{\frac{0}{0}}$ & $\begin{array}{l}\text { Ensaio Randomizado controlado. } \\
390 \text { grávidas em trabalho de parto vaginal foram divididas aleatoriamente em três grupos } \\
\text { (estimulação do mamilo, estimulação uterina, grupo controlo). Após o nascimento, os grupos } \\
\text { foram avaliados quanto à duração do nascimento e à indução sintética com oxitocina. }\end{array}$ \\
\hline 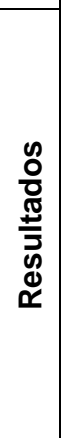 & $\begin{array}{l}\text { Relativamente à estimulação mamilar: } \\
\text { Outcome eficácia: O estudo estabeleceu diferenças estatisticamente significativas }(p<0,05) \text { entre } \\
\text { os grupos em termos de durações médias da primeira, segunda e terceira fases do TP, o rácio de } \\
\text { cesariana e a necessidade da indução do parto com ocitocia. As fases de trabalho de parto foram } \\
\text { mais curtas para o grupo de estimulação do mamilo (primeira fase: } 3,8 \text { horas, segunda fase: } 16 \\
\text { minutos, terceira fase: } 5 \text { minutos) quando comparado ao grupo controlo (primeira fase: } 6,8 \text { horas, } \\
\text { segunda fase: } 27 \text { minutos, terceira fase: } 6 \text { minutos). No grupo controlo, } 89,2 \% \text { das parturientes } \\
\text { foram submetidas à indução do parto e } 8,5 \% \text { a cesariana. Nenhuma mulher no grupo de } \\
\text { estimulação mamilar teve cesariana. } \\
\text { A estimulação mamilar reduz a frequência da indução eletiva do trabalho de parto, a taxa de } \\
\text { complicaçôes relevantes e promove o parto vaginal normal. Portanto, deve ser considerada para } \\
\text { mulheres grávidas em trabalho de parto }\end{array}$ \\
\hline
\end{tabular}

Study To Evaluate The Effectiveness of Nipple Stimulation For Progress of Labour During First Stage, Among Primigravida Mothers in Selected Hospital at Tirunelveli..

N.E.

Suja J./Tese de mestrado Nehru Nursing College/2015, Tirunelveli, Índia

\section{Ensaio Quasi-experimental.}

. 60 primigestas de termo, sem risco obstétrico, com início espontâneo do TP na primeira fase de

¿ TP, alocadas em 2 grupos: 30 no grupo experimental e 30 no grupo de controlo 


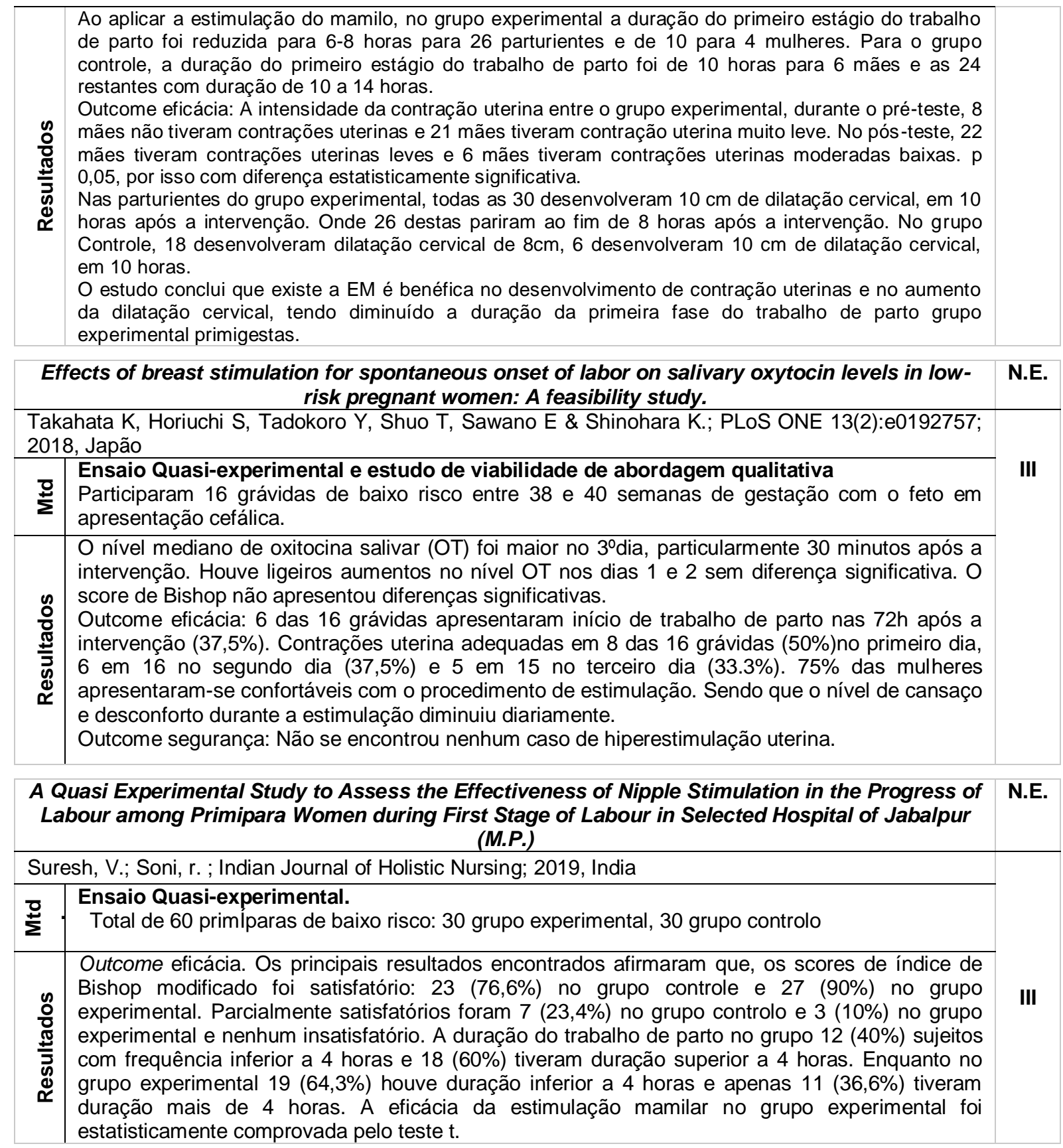

Após a análise dos estudos, constatámos que a amostra se concentrou em mulheres de termo com gravidez de baixo risco, à exceção da revisão de Razgaitis e Lyvers (2010) que não clarifica qual a população dos estudos selecionados. Pelo que, como refere o maior número dos investigadores; a maioria das conclusões apenas se podem debruçar sobre esta população. Relativamente à técnica de estimulação mamária, não existiu um consenso relativamente ao procedimento e/ou timing. Todavia, existe uma linha orientadora consensual de que a mama deve ser estimulada uma de cada, vez, diariamente, sendo que 
deve existir pausa e descanso entre cada estimulação (Demirel \& Guler, 2015; Kavanagh, Kelly \& Thomas, 2005; Razgaitis \& Lyvers, 2010; Singh et al., 2014; Suja, 2015; Suresh e Soni, 2019; Takahata et al., 2018). Para os ensaios analisados, identificamos e delineamos indicadores tradutores dos outcomes, eficácia e segurança, baseados nas diretrizes internacionais (ACOG, 2019; NICE, 2019, OMS, 2018). Para a eficácia: o índice de Bishop, o início do TP, cesariana e a satisfação da mulher; para a segurança alterações no traçado CTG (hiperestimulação uterina), a presença de mecónio e Apgar inferior a 7 aos 5 minutos, a necessidade de recorrer a cesariana pode ser também interpretada como um critério de segurança (Quadro 3).

Quadro 3 - Outcomes para os estudos

\begin{tabular}{|l|l|}
\hline \multicolumn{1}{|c|}{ Eficácia } & \multicolumn{1}{c|}{ Segurança } \\
\hline \hline - Índice de Bishop & $\begin{array}{l}\text { - Alterações no CTG } \\
\text { - Início de TP } \\
\text { - Satisfação da mulher } \\
\text { - Cesariana }\end{array}$ \\
\hline
\end{tabular}

OUTCOME SEGURANÇA

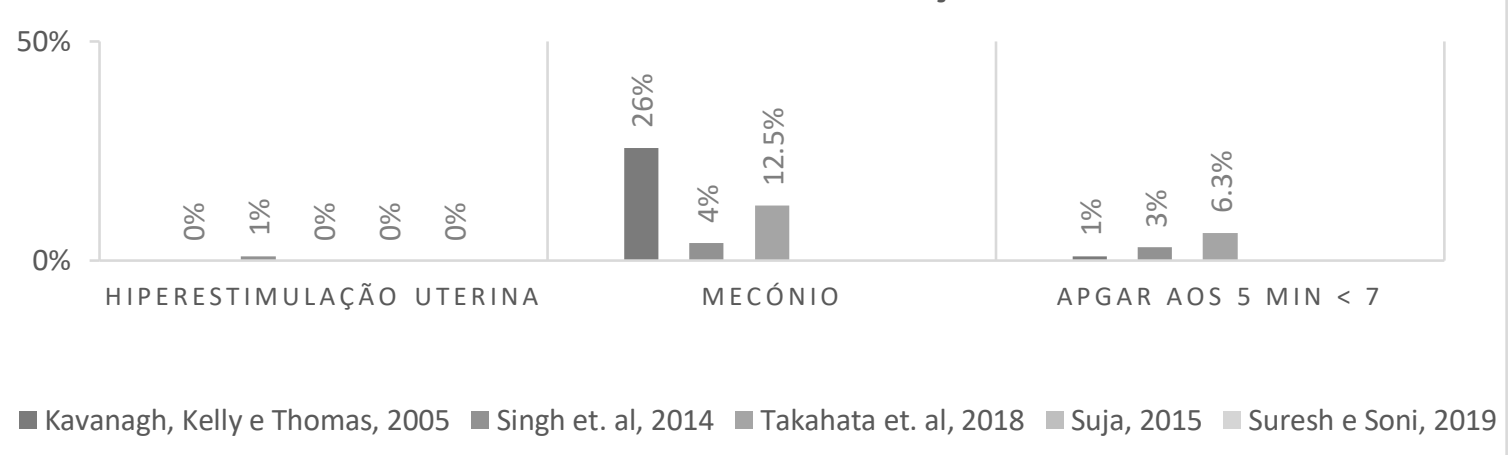

Figura 2- Outcome Segurança, Grávidas Baixo Risco

Centrando-nos na segurança, um dos riscos citados pelos autores relaciona-se com a possibilidade de hiperestimulação uterina, causada pela libertação não controlada de ocitocina pela EM, que, por sua vez, poderia colocar em causa o bem-estar fetal.

Nesse sentido, em quatro destes estudos foi avaliado a atividade uterina e o bem-estar fetal por CTG, assim como indicadores que pudessem traduzir sofrimento fetal; como a presença de mecónio à nascença e o índice de Apgar aos 5 minutos inferior a 7. Na revisão sistemática Cochrane ${ }^{\circledR}$ (Kavanagh, Kelly \& Thomas, 2005), na revisão de Razgaitis e Lyvers 
(2010), no estudo RTC de Singh et al., (2014) e no ensaio quase-experimental de Suresh e Soni (2019) não existiram diferenças

significativas relativamente à presença destes indicadores, denote-se, ainda, a ausência de casos hiperestimulação no estudo da Cochrane ${ }^{\circledR}$ (Kavanagh, Kelly \& Thomas, 2005) de Suja (2015), Suresh e Soni (2019) e de Takahata et al. (2018) (Figura 2). Contudo, quase todos os estudos, à exceção de Razgaitis e Lyvers (2010) que não clarifica a população, realçam para a segurança aplicada em grávidas de baixo risco, aconselhando a necessidade de estudos noutra população. Assim, a evidência disponível demonstra que a EM é um método seguro na indução de TP em grávidas de termo com gravidez de baixo risco. Relativamente ao outcome eficácia, percebemos que os estudos se mostram consensuais. Na maioria dos casos, as mulheres exibiram inícios de trabalhos de parto espontâneos, sendo de salientar a disposição para o seu início até 72h, como indica a revisão Kavanagh, Kelly e Thomas (2005) para a Cochrane ${ }^{\circledR}$. Por outro lado, apercebemo-nos que a falha deste método como indutor, não foi estatisticamente significativa, pelo que o recurso à indução sintética, como a cesariana foram menores que nos grupos de controlo (sem intervenção). No que concerne às alterações do índice de Bishop aparentemente não existiram diferenças estatisticamente significativas.

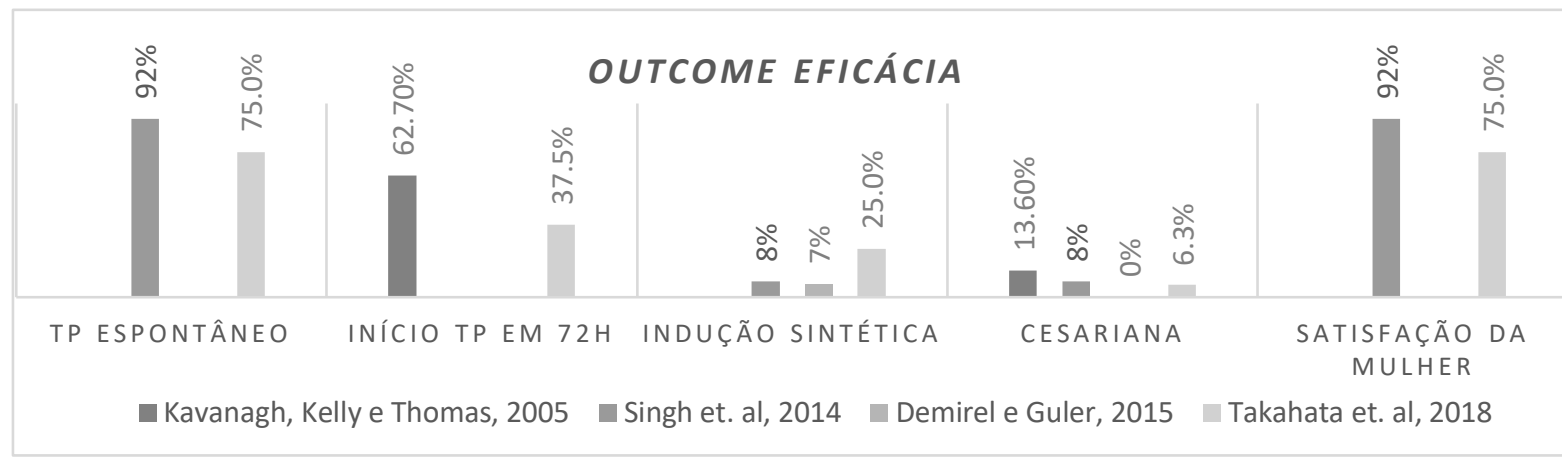

Figura 3 - Outcome Eficácia, Grávidas de Baixo Risco

Este dado poderá estar relacionado com o facto de as primíparas apresentarem piores condições do colo que as multíparas. No entanto, no ensaio de Demirel e Guler (2015), o grupo EM apresentou scores de índice de Bishop significativamente melhores que o grupo de controlo e, o de Suja (2015), em que as parturientes do grupo experimental apresentaram melhorias na dilatação cervical (Demirel \& Guler, 2015; Kavanagh, Kelly \&Thomas, 2005; Singh et al., 2014; Suja, 2015; Suresh e Soni, 2019; Takahata et al., 2018). 
Denote-se ainda, que os resultados de Demirel e Guler (2015), Suresh e Soni (2019) e de Suja (2015) cujo estudo apontou para o efeito positivo deste método, na aceleração das fases do trabalho de parto, nomeadamente, na redução da primeira (Suja, 2015; Suresh e Soni, 2019). Acresce ainda, o nível de aceitação e satisfação das mulheres com o procedimento, nos estudos de Demirel e Guler (2015) e de Tanaka et al. (2018) em que apresentaram taxas de $92 \%$ e $75 \%$ respetivamente (Figura 3). Assim, a evidência disponível demonstra que a EM é um método eficaz na indução de trabalho de parto em grávidas de termo com gravidez de baixo risco.

\section{CONCLUSÃO}

De acordo com os resultados dos estudos analisados, a estimulação mamária pode ser um método seguro e eficaz, sendo razoável considerá-la como uma opção viável para a indução do trabalho de parto para mulheres primíparas e multíparas, com gestações de baixo risco, que se sintam confortáveis a utilizar este método.

Enquanto método indutor, a evidência disponível suporta a EM como meio natural, não farmacológico, de amadurecimento do colo uterino, estimulador de contrações uterinas eficazes. Também de início do TP, associado a excelentes níveis de satisfação por parte da mulher e, ainda, reduz os rácios de hemorragia pós-parto. No entanto, relativamente à segurança do método, alguns autores alertaram para possíveis riscos associados à EM coligados à quantidade de ocitocina, não controlada, que é libertada durante a estimulação, nomeadamente, a hiperestimulação uterina. Todavia, a evidência disponível não vai de encontro a esta preocupação, demonstrando que o método se mostra seguro, mas, que pela falta de mais estudos noutra população, indicado apenas para mulheres com gravidez de baixo risco.

A EM pode apresentar -se como solução natural, não-farmacológica, em caso de indução de trabalho de parto, como no caso de grávidas de termo, com 41 semanas de gestação completas. É, no entanto, importante salientar que este método como indutor do TP não é tão rápido quanto o farmacológico, pelo que é importante delinear corretamente o timing para iniciar a intervenção. Relativamente à forma de implementação, existe uma variedade de técnicas descritas nos estudos, mas os investigadores são concisos e aconselham a estimulação bilateral, que a grávida se foque numa mama de cada vez, intercalando períodos de descanso. 
Após finalizarmos este estudo, concluímos que a metodologia adotada foi a mais adequada, em consonância com o objetivo e questão de investigação. Esta permitiu-nos identificar, não só o estado de arte, neste domínio do conhecimento sobre o método da EM, como também, evidenciar as suas limitações. Identificamos como principal limitação a escassez de estudos de investigação no âmbito dos métodos não farmacológicos indutores de TP. Concretamente da estimulação mamária, assim como, estudos que abordem a segurança e satisfação deste método em grávidas de risco centrados em estudos de abordagem qualitativa.

Realçamos, ainda a pertinência desta metodologia face aos resultados e recomendações obtidos, que nos permitem dar a sugestão para a realização de outras investigações de domínio qualitativo. Nomeadamente, para compreender o significado que as mulheres atribuem à utilização deste método. Esperamos, ainda, que este trabalho opere como alavanca, para impulsionar investigações qualitativas que problematizem os métodos indutores do trabalho de parto, que incluam a perspetiva da mulher relativamente à sua eficácia e satisfação, para que de uma forma indutiva, possam emergir dados que constituam uma mais valia para delinear intervenções que possam ir ao encontro da singularidade de cada mulher e de cada indução de trabalho de parto.

Nesta linha de entendimento, relativamente às implicações para a prática, este estudo pode, ainda, contribuir para consciencializar os enfermeiros especialistas em saúde materna e obstétrica, para a importância da sua autonomia na ação. Também, para a responsabilidade de prestar cuidados de qualidade congruentes, seguros e eficazes, ao desenvolver intervenções promotoras de contrações uterinas, de acordo com o método de indução, escolhido pela mulher, para iniciar o seu trabalho de parto. Pode, desta forma, demonstrar a sua competência cultural, ao respeitar os valores e as crenças da pessoa, ou seja; a sua cultura e o seu estatuto sociocultural.

Por fim, consideramos que esta investigação permite incitar ao desenvolvimento do conhecimento sobre métodos naturais associados à indução e ao caminho para um parto normal, em situações de baixo risco, contribuindo, assim, para o progresso do conhecimento e, consequentemente, para uma Enfermagem Avançada em cuidados especializados.

\section{REFERÊNCIAS}

ACOG (2019)- Clinical management guidelines for obstetrician-gynecologists Practice Bulletin Induction of Labor 2009 reaffirmed 2019. Obstetrics \& Gynecology. number 107, p.386- 397 
Demirel, G. \& Guler, H. (2015). The Effect of Uterine and Nipple Stimulation on Induction With Oxytocin and the Labor Process. Worldviews on Evidence-Based Nursing. 12:5, 273-280

Kavanagh J, Kelly Aj, Thomas J. (2005). Breast stimulation for cervical ripening and induction of labour. Cochrane Database of Systematic Reviews, Issue 3. Art. No.: CD003392.

McCormack, B.; Cardiff, D.; Jacobs, J.; Karlsson, M.; Mekki, P. \& Lieshout, W..(2015) Person-centredness - the 'state' of the art. International Practice Development Journal, 5 (1), 1-15

Mendes, K. D. S.; Silveira, R. C. C. P. \& Galvão, C. M. (2008) Revisão integrativa: método de pesquisa para a incorporação de evidências na saúde e na enfermagem. Texto Contexto Enfermagem, Florianópolis. Vol. 17, no 4. out/dez. p. 758-764

NICE (2018). Induction of labour. NICE clinical guideline 702008 reviewed 2018. UK.

Organização Mundial de Saúde (2018). WHO recommendations: intrapartum care for a positive childbirth experience. Geneva: World Health Organization. License: CC BY-NC-SA 3.0 IG

Razgaitis J \& Lyvers, A. (2010). Management of Protracted Active Labor with Nipple Stimulation: A Viable Tool for Midwives? J Midwifery Womens Health; vol 55. P. 65-69.

Singh N, Tripathi R, Mala YM \& Yedla N. (2014). Breast Stimulation in Low-Risk Primigravidas at Term: Does It Aid in Spontaneous Onset of Labour and Vaginal Delivery? A Pilot Study. BioMed Research International. Vol 1. PP 1 - 6. Article ID 695037.

Sousa L., Vieira, C., Severino, S. \& Antunes, A.(2017). A metodologia de revisão integrativa da literatura em enfermagem. Revista Investigação em Enfermagem. Novembro: 17-26

Suja, J (2015) A study to evaluate the effectiveness of nipple stimulation for progress of labour during first stage, among primigravida mothers in a selected hospitals at Tirunelveli, Tamil Nadu. Tese de Mestrado, Nehru Nursing College, India - Tirunelveli.

Suresh V. e, Soni R. (2019). A Quasi-Experimental Study to Assess the Effectiveness of Nipple Stimulation in the Progress of Labour among Primípara Women during First Stage of Labour in Selected Hospital of Jabalpur (M.P.). Ind J Holist Nurs; 10(1): 1-7.

Takahata K, Horiuchi S, Tadokoro Y, Shuo T, Sawano E \& Shinohara K. (2018). Effects of breast stimulation for spontaneous onset of labor on salivary oxytocin levels in low-risk pregnant women: A feasibility study. PLoS ONE 13(2): e0192757.

Whittemore R. \& Knafl K. (2005) The integrative review: updated methodology. Journal of Advanced Nursing 52(5), 546-553. 